\title{
A Plugin Architecture for the TAU Performance System
}

\author{
Allen D. Malony \\ University of Oregon \\ Eugene, Oregon \\ malony@cs.uoregon.edu
}

\author{
Srinivasan Ramesh \\ University of Oregon \\ Eugene, Oregon \\ sramesh@cs.uoregon.edu
}

\author{
Kevin Huck \\ University of Oregon \\ Eugene, Oregon \\ khuck@cs.uoregon.edu
}

\author{
Nicholas Chaimov \\ ParaTools, Inc. \\ Eugene, Oregon \\ nchaimov@paratools.com
}

\author{
Sameer Shende \\ University of Oregon \\ Eugene, Oregon \\ sameer@cs.uoregon.edu
}

\begin{abstract}
Several robust performance systems have been created for parallel machines with the ability to observe diverse aspects of application execution on different hardware platforms. All of these are designed with the objective to support measurement methods that are efficient, portable, and scalable. For these reasons, the performance measurement infrastructure is tightly embedded with the application code and runtime execution environment. As parallel software and systems evolve, especially towards more heterogeneous, asynchronous, and dynamic operation, it is expected that the requirements for performance observation and awareness will change. For instance, heterogeneous machines introduce new types of performance data to capture and performance behaviors to characterize. Furthermore, there is a growing interest in interacting with the performance infrastructure for in situ analytics and policybased control. The problem is that an existing performance system architecture could be constrained in its ability to evolve to meet these new requirements. The paper reports our research efforts to address this concern in the context of the TAU Performance System. In particular, we consider the use of a powerful plugin model to both capture existing capabilities in TAU and to extend its functionality in ways it was not necessarily conceived originally. The TAU plugin architecture supports three types of plugin paradigms: EVENT, TRIGGER, and AGENT. We demonstrate how each operates under several different scenarios. Results from larger-scale experiments are shown to highlight the fact that efficiency and robustness can be maintained, while new flexibility and programmability can be offered that leverages the power of the core TAU system while allowing significant and compelling extensions to be realized.
\end{abstract}

\section{KEYWORDS}

parallel performance, measurement, runtime analytics, plugin

Permission to make digital or hard copies of all or part of this work for personal or classroom use is granted without fee provided that copies are not made or distributed for profit or commercial advantage and that copies bear this notice and the full citation on the first page. Copyrights for components of this work owned by others than ACM must be honored. Abstracting with credit is permitted. To copy otherwise, or republish, to post on servers or to redistribute to lists, requires prior specific permission and/or a fee. Request permissions from permissions@acm.org.

ICPP 2019, August 5-8, 2019, Kyoto, Japan

(C) 2019 Association for Computing Machinery.

ACM ISBN 978-1-4503-6295-5/19/08_..\$15.00

https://doi.org/10.1145/3337821.3337916

\section{ACM Reference Format:}

Allen D. Malony, Srinivasan Ramesh, Kevin Huck, Nicholas Chaimov, and Sameer Shende. 2019. A Plugin Architecture for the TAU Performance System . In 48th International Conference on Parallel Processing (ICPP 2019), August 5-8, 2019, Kyoto, Japan. ACM, New York, NY, USA, 11 pages. https: //doi.org/10.1145/3337821.3337916

\section{INTRODUCTION}

The advances in high-performance computing (HPC) hardware and systems have made it possible to develop parallel applications of greater sophistication and power for purposes of achieving more ambitious objectives in computational and data science domains. With the potential for scalable parallelism, heterogeneous execution, massive concurrency, and low-latency/high-bandwidth interconnection, the challenge for applications is how to maximize the advantage these advances bring. Clearly, the evolution of HPC technology and integration have increased the complexity of this challenge. While new features in parallel languages, programming tools, and runtime system environments can help to transform existing applications or to develop new ones in ways that leverage HPC's strengths, they can also introduce complexities of their own. At the end of the day, the goal of gaining high performance is paramount, but productivity and performance portability concerns are important as well.

Throughout the history of parallel computing, it has been important to characterize and understand the performance of HPC systems and the applications that run on them. For this purpose, parallel performance systems have been developed to empirically analyze real applications on real machines. Several robust performance systems have been created for parallel systems with the ability to observe diverse aspects of application execution on the different underlying hardware. A key objective is to support measurement methods that are efficient, portable, and scalable. For these reasons, the performance measurement infrastructure is tightly embedded with the application code and runtime execution environment. Unfortunately, the performance system is not immune to the changes in HPC system environment and parallel programming methodologies. Researchers in the parallel tools' community know very well the constant attention needed to massage and extend a performance system to support the rich space of possible parallel operation that it is supposed to observe.

Indeed, it is remarkable that these parallel performance systems can do what they do on leading HPC platforms and for the variety of applications run on them today. However, as HPC systems and 
parallel software evolve, especially towards more heterogeneous, asynchronous, and dynamic operation, it is to be expected that the requirements for performance observation and awareness will change. For example, there is a growing interest in interacting with the performance infrastructure for in situ analytics and policy-based control. The problem is that the performance systems may need to react to new demands for performance observation that might go beyond the core features of the performance system. More seriously, the performance system architecture could be constrained in its ability to evolve to meet these new requirements.

Such is the case in this paper. Here we report our research efforts to address these type of evolutionary concerns in the context of the TAU Performance System. Given the goals of TAU to support current and next-generation high-performance parallel applications, it is necessary to create mechanisms to enable advanced performance measurement and analysis solutions. Specifically, we consider the use of a powerful plugin model to both enhance TAU's existing capabilities, and to extend its functionality in ways it was not necessarily conceived originally. The TAU plugin architecture supports three types of plugin paradigms: EVENT, TRIGGER, and AGENT. We demonstrate how each operates under several different scenarios. Results from large-scale experiments are shown to highlight the fact that efficiency and robustness can be maintained, while new flexibility and programmability can be offered that leverages the power of the core TAU system while allowing significant and compelling extensions to be realized.

We begin with an overview of TAU's current design and implementation in Section $\S 2$. This will set the stage for the plugin architecture proposed in Section §3. Here we also describe the implementation of the TAU plugin prototype. In Section §5, we give demonstrations of its use. More interesting experiments are shown in Section §6. Other performance systems have addressed related issues. We discuss those research efforts in Section §7. Finally, the paper outlines future work and gives concluding remarks.

\section{BACKGROUND}

\subsection{TAU Overview}

The TAU Performance System ${ }^{\circledR}$ [1] is the product of $25+$ years of development to create a robust, flexible, portable, and integrated framework and toolset for performance instrumentation, measurement, analysis, and visualization of large-scale parallel computer systems and applications. TAU supports all major parallel paradigms (shared memory multithreading, distributed memory message passaging, data parallel acceleration), parallel programming models (e.g., MPI, OpenMP, OpenACC), and languages (e.g., C, C++, Fortran, Python). The TAU software is open source and has been ported to many processor architectures and HPC platforms around the world. It has been utilized in many performance analysis and optimization efforts across a wide spectrum of science and engineering applications.

From TAU's perspective, the execution of a program is regarded as a sequence of significant performance events. TAU was originally conceived to observe these events through probes inserted in the application code. The combination of a flexible event model and multiple instrumentation techniques allowed TAU to effectively morph its observation capabilities to captures events and their semantics that might otherwise be intractable. Over time, TAU expanded its observation approach to include event-based sampling (EBS) methods [2], where the "event" here is an interrupt to the application's execution. TAU's EBS support adds performance observability and detail. Both probes and statistical sampling (i.e., EBS) can be used simultaneously in TAU.

Logically, once "events" are made visible (via probes or sampling) they can be measured. The TAU measurement system event interface allows events to be defined, their visibility controlled, and their runtime data structures to be created. Each event has a type (atomic or interval), a group, and a unique event name. The event name is a character string and is a powerful way to encode event information. At runtime, TAU maps the event name to an efficient event ID for use during measurement. Events are created dynamically in TAU by providing the event interface with a unique event name. This makes it possible for runtime context information to be used in forming an event name (context-based events), or values of routine parameters to be used to distinguish call variants, (parameter-based events). TAU also supports phase and sample events.

The purpose of event control in TAU is to enable and disable a group of events at a coarse level. This allows the focus of instrumentation to be refined at runtime. All groups can be disabled and any set of groups can be selectively enabled. Similarly, all event groups can be enabled initially and then selectively disabled. It is also possible to individually enable and disable events. TAU uses this support internally to throttle high overhead events during measurement.

The measurement system is the heart and soul of TAU. It has evolved over time to a highly robust, scalable infrastructure portable to all HPC platforms. The instrumentation layer defines which events will be measured and the measurement system selects which performance data metrics to observe. Performance experiments are created by selecting the key events of interest and by configuring measurement modules together to capture desired performance data. TAUś measurement system provides support for portable timing, integration with hardware performance counters (e.g., PAPI [3]), parallel profiling, parallel tracing (with OTF-2 [4]), and runtime monitoring.

TAU's measurement system has two core capabilities. First, the event management handles the registration and encoding of events as they are created. New events are represented in an event table by instantiating a new event record, recording the event name, and linking in storage allocated for the event performance data. The event table is used for all atomic and interval events regardless of their complexity. Event type and context information are encoded in the event names. The TAU event management system hashes and maps these names to determine if an event has already occurred or needs to be created. Events are managed for every thread of execution in the application.

Second, a runtime representation called the event callstack captures the nesting relationship of interval performance events on each thread. It is a powerful runtime measurement abstraction for managing the TAU performance state for use in both profiling and tracing. In particular, the event callstack is key for managing execution context, allowing TAU to associate this context with the events being measured.

The final component of TAU is analysis tools. TAU includes support for parallel profile data management 
(TAUdb [5]), analysis(ParaProf [6]), and data mining (PerfExplorer [7]). It leverages existing trace analysis functionality available in robust external tools, including the Vampir [8], Jumpshot [9], and Expert/CUBE $[10,11]$.

\subsection{TAU Operation}

Given this overview, let us consider how the TAU measurement actually operates. The TAU measurement system is implemented as a library that is loaded with a parallel application and lives within each application process on every node that is allocated to the job. During execution, events occurring within threads of execution on a process are then measured by TAU and stored in thread-specific data structures. That is to say, if a parallel profile measurement is being made, each application thread of execution will have its own profile data for the events that occurred on that thread. Similarly, each application thread will output a separate trace of events from that thread. TAU collects a variety of metrics for events instrumented by probes and/or captured by event-based sampling in parallel profiles and/or traces. All of the TAU performance data is stored in the memory space of each process.

Interval events capture performance metrics that occur between entry and exit actions. The metrics could include execution time, hardware counters, or software counters. Atomic events are used to capture metrics of interest at a particular point in time or location in the code. Metrics could include hardware counters, message sizes involved in MPI communication, memory allocation, and so on. For every thread of execution within a process, TAU collects data for every interval and atomic event instrumented.

Figure 1 shows the two event types occurring on a thread of execution and TAU's updating of the thread's performance data structure. Figure 2 portrays how TAU operates for multiple application processes. All of the TAU performance data measured for each process thread is stored in the process memory space. Because TAU is a library, it will run within the process thread that called it. For this reason, measurement should be made as efficiently as possible so TAU can return to the application quickly. Furthermore, TAU is a thread-safe library and can be running simultaneously on each application thread of execution. In a distributed MPI application, TAU will generate performance data that resides locally within each MPI rank.

TAU measurements are recorded in parallel profiles and/or traces. Figure 3 portrays the profiles kept for all application processes which are spread across the HPC nodes allocated for the execution. TAU collects these profiles at the end of program execution and saves the performance information for offline analyses. TAU provides a routine for a process to access its thread profiles during execution, including taking a snapshot of the entire profile data.

\subsection{Constraints}

While TAU offers powerful measurement capabilities which have been successfully applied over many years, there are aspects of its present operation that will impose constraints to its use in the future. These include:

- All TAU execution takes place on application threads, including event measurements and interrupt handlers. There is no

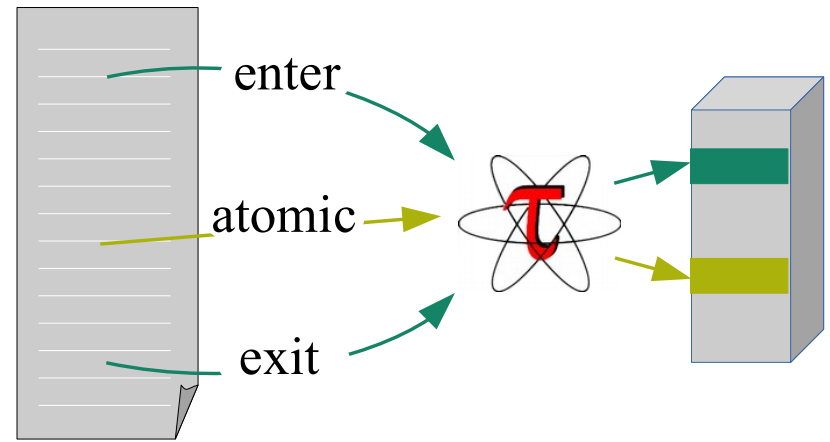

Figure 1: TAU supports interval and atomic events. Event measurements are made for each event occurring on each thread of execution. The performance data is store in a thread-specific data structure.

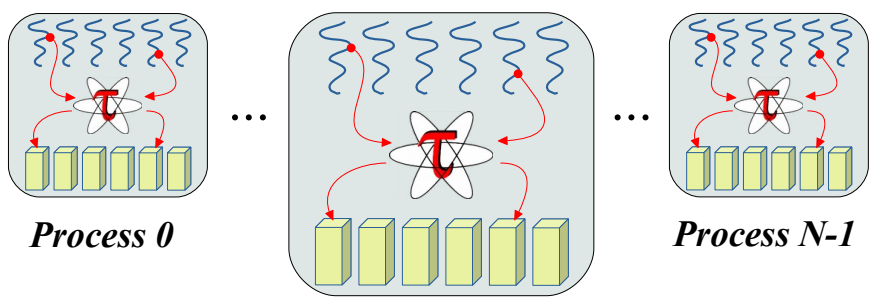

Figure 2: TAU captures performance data for all threads in each application process.

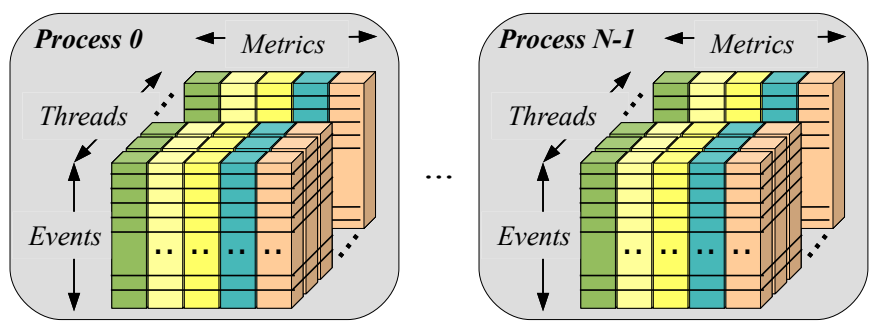

Figure 3: TAU collects parallel profiles for different events across all threads and processes in an application's execution. The "global" parallel profiles are distributed across all nodes of the application.

support for TAU-only thread resources to aid in any runtime tasks.

- TAU measures exactly the same metrics for all interval events. It is not possible, for example, to measure only time for one event and only cache misses for another event.

- If TAU is set up for profiling and tracing, all events are profiled and all events are traced. It is not possible to have only a subset of events traced.

- Although TAU offers "user-defined" events (both interval and atomic), the application can not pass TAU any data. The 
atomic interface has basic support for processing application values, but the functionality is limited.

- TAU resides in each application process and thus executes in a distributed manner, but there is no support for TAU to interact across the other processes except at the end of the program.

- Beyond the EBS support in TAU, there are rudimentary mechanisms for handling signals and asynchronous events.

Our objective is to update and extend the TAU architecture in such a way to address these concerns. We believe that doing so will "open up" TAU development to enhancements with broad interest.

In general, TAU is not dissimilar to other major parallel performance system in its design and operation, including Score-P [12], HPCToolkit [13], Scalasca [14], Vampirtrace [8], Extrae [15], and Open|SpeedShop [16]. It is possible that these performance systems struggle with the same limitations.

\section{TAU PLUGIN ARCHITECTURE}

Our objective is to overcome the potential future constraints to TAU by updating its system design architecture to allow new functionality to be developed. Specifically, we look to a plugin architecture to extend the core measurement and analysis capabilities in TAU. The general idea is that a plugin will register callbacks to respond to certain states of TAU execution. When such a TAU state occurs, any registered plugins are called with data appropriate for the state semantics. In this manner, the plugin operation can be informed of its context and implement additional processing. Below we discuss our design and development approach to a TAU plugin architecture.

Of course, the challenge for a performance system such as TAU with mature capabilities in frequent use is how to "integrate" a plugin architecture into an existing implementation. The tension between retrofitting an existing system and wholesale redesign is always there. Nevertheless, the plugin architecture we hope for should be designed to address as best as possible the following criteria:

- Separation of concern between what action invokes a plugin and what functionality the plugin provides.

- Control over enabling/disabling of plugin invocation versus control of plugin operation.

- Access to TAU's internal operations and performance information.

- Flexible plugin programming methods that are not constrained by necessarily by TAU development rules.

These criteria are better thought of as guidelines for the plugin architecture rather than strict requirements, since they will be addressed at different degrees depending on what is being done.

The following describes the approach taken for our prototype TAU plugin architecture. We begin with a description of the "state classes" distinguished by TAU and what additional access points might be relevant. We then discuss the operational model for plugins at a high level, followed by a description of the plugin prototype implementation.

\subsection{TAU States and STATE Plugins}

TAU supports multiple capabilities and is in different states of processing depending on what it is doing at a particular time. For instance, Section $\S 2$ describes at a high-level what happens when TAU makes measurements for interval and atomic events, but there are other functionalities that TAU supports that are relevant to identify. The general idea is that plugins could register callbacks to one or more of TAU's "states" of execution that could be "salient" or "interesting" from a perspective of additional plugin processing.

Presently, TAU recognizes several states of execution that effectively correspond to locations inside the TAU performance system where the processing is taking place. Each state will have a statespecific context that captures relevant information of importance of the plugin. If the state context identifies the particular instance, we will say that the plugin is named, otherwise, it is generic. For example, when TAU is in a FUNCTION_ENTRY processing state, the name of the function being entered is part of the state context, making it possible for the plugin to be invoked with the function name, to distinguish it from other occurrences of FUNCTION_ENTRY processing. Below is a non-exhaustive list of TAU states that are presently available for plugin support.

- FUNCTION_ENTRY/EXIT: When TAU measures interval events, the profiling module is invoked at the entry and exit of every instrumented interval event. The FUNCTION_ENTRY and the FUNCTION_EXIT TAU states occur accordingly. Here, the interval event name, TAU's associated function ID, function group, and timestamp information is context data that can be passed to a registered named plugin.

- PHASE_ENTRY/EXIT: TAU makes it possible to demarcate phases within the application source code. The PHASE_ENTRY and PHASE_EXIT TAU states represent the TAU processing states specific to phases. The phase name is a part of the context provided to a registered named plugin for invocation with every entry/exit of static and dynamic phases.

- INTERRUPT_TRIGGER: TAU installs a signal handler for the SIGUSR signal to implement sampling operations. When sampling is turned on and TAU's signal handler is run, TAU is in the INTERRUPT_TRIGGER state. Different interrupt intervals are possible. Any registered plugins are invoked with a generic context.

- MPI_T: When TAU is configured to support the MPI Tools Information Interface (MPI_T [17]), TAU collects performance variable data (PVARs) through the MPI_T interface. Each PVAR has a name and a unique ID associated with it. When TAU is querying a PVAR value, it is in the MPI_T state. Registered named plugins can be called with the PVAR name and ID.

- POST_INIT/PRE_END_OF_EXECUTION: During its initialization phase, TAU performs a number of important internal tasks such as invoking the init() routines of downstream APIs, allocating memory for timers, and so on. TAU is in the POST_INIT state then. Similarly, during its finalization phase after profiling has been completed, TAU frees up allocated memory, invokes the finalize() routines of APIs, and prepares to shut down operations and write out the profile or trace information that has been collected. TAU is in the PRE_END_OF_EXECUTION state then. During these states, registered generic plugins can utilize TAU support to perform specialized tasks that rely on parallel programming 

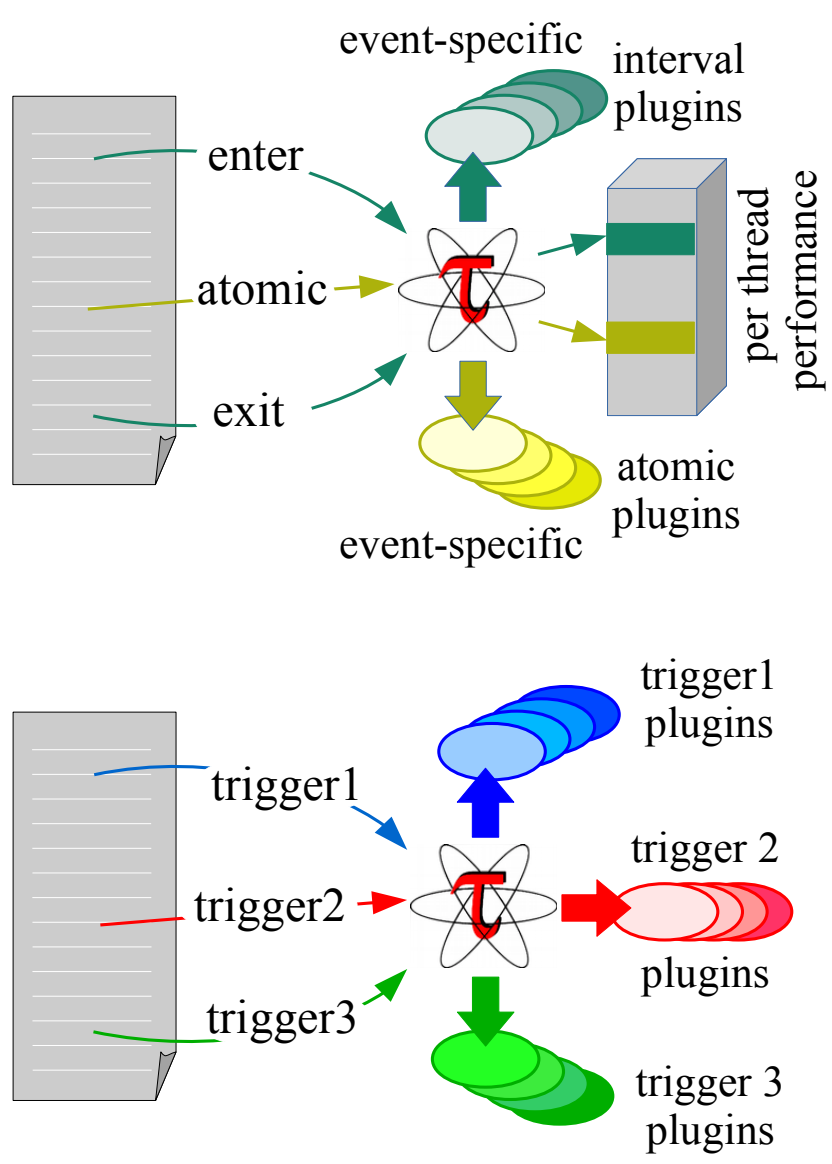

Figure 4: (Top) STATE plugin operation for interval and atomic events. (Bottom) TRIGGER plugin operation.

and profiling libraries to be in a certain state (initialized or finalized).

We have designed STATE plugin support to work with TAU states and have developed specific plugins for each of the cases above. Figure 4 (top) graphically portrays how the plugins work for the FUNCTION_ENTRY/EXIT and ATOMIC TAU states. Because this is a plugin for a named state, it is called with an interval event name or atomic event name, plus other context information. The plugin can then process the event with or without specializing its function based on the context. Event-specific plugin processing is indicated by the plugin shading. Notice, the standard TAU performance measurements are still being performed. The plugin processing is whatever the plugin developer implemented.

\subsection{Beyond TAU and TRIGGER Plugins}

TAU states discussed above are all defined by and occur within the TAU performance system. Registering generic and named plugins for TAU states offers a clear separation of concern from the state occurrence and any plugin action associated with it, beyond the standard TAU measurement processing. In essence, it allows for enhanced observation and actuation of extended functionality "triggered" by TAU state. We will refer to these plugins as TRIGGER plugins.

Should it be the case that only internal TAU processing can trigger plugins? Consider a situation where the application desires an analysis of current TAU performance data at a particular point in its execution. For instance, it might be interested in detecting performance anomalies per iteration of a time-stepped simulation on a large HPC system. One example of a performance anomaly is a sudden increase in communication time that deviates significantly from regular behavior. If such an anomaly is detected, the application could decide to checkpoint certain aspects of its application state, as well as performance state, for offline analysis. The analysis task can be broken down into the following distinct elements:

- The application invokes (somehow) performance processing within TAU at the end of every iteration.

- Communication performance is aggregated and stored in a buffer.

- Anomalies are analyzed with respect to previously stored data.

- Results are returned (somehow) to the application.

How should this functionality be developed? It seems reasonable to want to actuate certain operations for a task like this independent of a particular TAU state. In fact, it should be expected that the "triggering" of these operations could occur outside of TAU altogether, such as from the application itself. However, it is still important that the operations run "inside" the TAU environment so that they can access the TAU performance data.

In keeping with our plugin design concerns, we want to register a plugin with respect to a "trigger" that actuates the plugin when "fired" while keeping plugin functionality isolated within its implementation. To do so, a means to create a trigger, to fire the trigger, and receive a result is required. We have designed the TRIGGER plugin architecture that does just that. Like the STATE plugin, a TRIGGER plugin will run on an application thread of execution in the TAU environment with access to TAU performance data. However, it requires a new API to create and fire the trigger and exchange data across the interface. Figure 4 (bottom) shows how the TRIGGER plugins operate. They are named in the sense that a trigger has a unique name and plugins register for specific triggers.

\subsection{AGENT Plugins}

Both STATE plugins and TRIGGER plugins are synchronous in that they are invoked by TAU run on the same application thread as TAU, and are expected to return to TAU. Interestingly, TAU was originally designed to execute on the same threads of execution as the application. For the most part, it continues to do so now. Suppose that we relax this constraint and allow TAU to create new threads that then run in an asynchronous manner to the application. The concept of an AGENT plugin is based on exactly this thought. It is a plugin that has its own thread resources to do things that are not otherwise bound by application or TAU processing. Its functionality benefits from running on a thread within the process and hence has full access to TAU information. Like STATE and TRIGGER plugins, in fact, it can allocate memory for its operation. It can interact (for the purpose of exchanging data or control) using 
thread synchronization mechanisms with the TAU system and with other plugins.

\section{PLUGIN IMPLEMENTATION}

The plugin system is implemented in $\mathrm{C}++$ with $\mathrm{C} / \mathrm{C}++$ interfaces. The user can specify the path to the directory containing the plugins using the environment variable TAU_PLUGINS_PATH. The user can also specify the plugins to be loaded using the environment variable TAU_PLUGINS, separating the plugins by use of a delimiter.

The plugin system in TAU operates through the following phases:

- Initialization: This is invoked during TAU library initialization. During this phase, TAU's plugin manager reads the environment variables

TAU_PLUGINS_PATH and

TAU_PLUGINS and loads the plugins. Internally, each loaded plugin is associated with a unique, unsigned integer ID for the duration that it is in scope. Each plugin must implement a function called

Tau_plugin_init_func. Inside this function, every STATE plugin registers callbacks for a subset of TAU states.

- Callback: When TAU states occur during execution, the plugin manager invokes any registered callbacks for the specific TAU state in the increasing order of their ID's. Each state that is supported has a specific, typed data object associated with it. When the state occurs, this data object is populated and sent as a parameter to the plugin callback. The TRIGGER plugin is special as it serves the purpose of analyzing application-defined data. As a result, a void ${ }^{*}$ pointer is passed to such plugins from within the application. It is up to the plugin developer (user) to know how to decipher this information from within the plugin.

- Finalize phase: When TAU is done processing the performance data measured for an application, the plugins are notified to complete their operation. They are then unloaded, and all the auxiliary memory resources allocated by the plugin manager are freed. Optionally, the plugin may use this phase to write out any performance data for offline analysis at the end of the execution.

4.0.1 Enabling Customizability and Runtime Control. Section 3.1 introduces the notion of named and generic states in TAU. For instance, the function name field is used to distinguish between the various FUNCTION_ENTRY states. The entry of functions foo() and bar () represent separate, distinguishable states in our framework.

By default, all registered plugins for a given state are invoked when the state occurs. We define customizability as the ability to specify the execution of a subset of all registered plugins on the occurrence of a certain named state. Customizability is valid only for named states. Internally, named states are hashed based on the name field and a map is used to store the list of plugins to execute for the specific named state. TAU allows users to modify this map through the plugin API. Note that the full name of the state or its regular expression can be used to access the map.

We define runtime control as the ability to enable or disable specific plugins for specific states (named or generic) at runtime. The TAU plugin API allows users access to such functionality. This API requires the name of the event (or its regular expression) along
Table 1: LULESH: Processing state counter values on thread 0, MPI rank 0 (other ranks also captured)

\begin{tabular}{|l|c|}
\hline Processing State & Counter Value \\
\hline FUNCTION_REGISTRATION & 29 \\
FUNCTION_ENTRY & 23528 \\
FUNCTION_EXIT & 23538 \\
ATOMIC_EVENT_REGISTRATION & 2 \\
ATOMIC_EVENT_TRIGGER & 500 \\
\hline
\end{tabular}

with the plugin ID. The same functionality is extended to trigger states as well, where the trigger ID is used in place of the name. We envision runtime control to be invoked infrequently in the program, such as when a phase change occurs. Also, we expect both customizability and runtime control to be invoked in a serial portion of the program. These semantics allow for a simpler plugin framework design that involves a significantly reduced need for locking of internal data structures.

\section{USAGE SCENARIOS}

In this section, we describe some of the scenarios that motivate and are enabled by the design of our plugin system.

\subsection{Event Counter}

In order to broadly demonstrate our plugin system, we designed a plugin that registers callbacks for a variety of supported TAU plugin processing events. The events of primary interest to us are FUNCTION_ENTRY, FUNCTION_EXIT, and ATOMIC_EVENT_TRIGGER. Inside the callback for the plugin event, a thread-level counter keeps track of the number of times that TAU has seen that event.

No filtering of any sort is performed on the processing state: the plugin is invoked for every named and generic processing state encountered. For example, the FUNCTION_ENTRY state is invoked at the entry of every function that is instrumented by TAU. The rationale behind designing this plugin is to correlate information collected by the plugin with the information collected by TAU's measurement system. Figure 5 a summarizes the number of function calls made by thread 0, MPI rank 0 of a LULESH [18] application that has been instrumented and built with MPI+OpenMP support. Figure $5 \mathrm{~b}$ depicts the number of atomic events that have been triggered on this thread. Table 1 is the statistics that are output by the event counter plugin for thread 0 , MPI rank 0 at the end of execution. It is evident that this correlates well with the profiling information collected by TAU, depicted in Figure 5.

\subsection{Selective Tracing}

Section 2 describes the types of events that TAU measures: interval and atomic events. Every event that passes through TAU's measurement system is either profiled and/or traced. As such, there is no mechanism in TAU that allows one to specify separate sets of events to profile and trace. In other words, the action to profile and/or trace is specified at a high level and is applied across the board for all instrumented events.

The plugin system allows one to separate the occurrence of an event from the action associated with it. Particularly, it allows for 


\begin{tabular}{|l|r|r|r|}
\hline File Options Windows Help & \multicolumn{2}{c|}{ NumSamples } \\
\hline Name & $\mathbf{3 , 9 9 2}$ & $\mathbf{4 9 9}$ \\
\hline Message size for all-reduce & Total & 1
\end{tabular}

(a) Atomic events

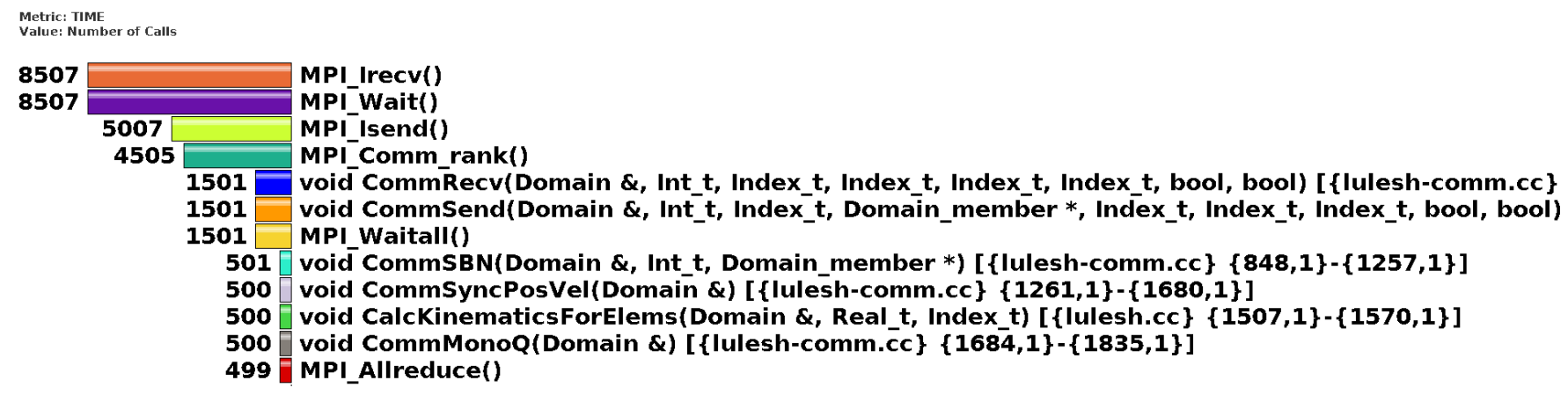

(b) Number of function calls

Figure 5: Paraprof: LULESH profile

the capability to profile all events while only tracing a subset of those events. This is useful in at least two ways:

- It reduces the size of the trace file generated.

- It reduces the amount of information in the trace file making it easier to visualize only the events that are important.

A plugin that performs selective tracing registers callbacks for the FUNCTION_ENTRY and FUNCTION_EXIT processing states and performs tracing inside these callbacks. The selection of these named processing states, however, is performed from within the application using TAU's plugin API. The application uses $\mathrm{C}++$ regex support to enable/disable selective tracing for classes of functions. In order to demonstrate the usage of this functionality, we consider a LULESH application that been instrumented with the Program Database Toolkit (PDT) and built with MPI+OpenMP support. We trace all events except MPI_Wait events. Figure 6a depicts the OTF2 trace visualization generated in Vampir [19] when all instrumented events are traced. Figure $6 \mathrm{~b}$ depicts the trace visualization generated when tracing for MPI_Wait events is selectively disabled using TAU's plugin infrastructure.

\subsection{Filter Plugin}

When instrumenting an application using compiler-based instrumentation or PDT, TAU uses a selective instrumentation file to selectively enable/disable instrumentation for functions and/or files. We developed a filter plugin that allows us to enable filtering at runtime. The plugin registers a callback for the FUNCTION_REGISTRATION_COMPL processing state that is invoked exactly once for every function that is instrumented using TAU. Inside the callback, the plugin checks the selective instrumentation file to see if instrumentation needs to be disabled for the function in question.

This functionality enables selective instrumentation to be performed without the need to recompile the application in order to perform different targeted performance studies. As opposed to the selective tracing plugin, the filter plugin enables specialization of plugin functionality through an external mechanism (selective instrumentation file).

\subsection{TAU SOS Plugin}

The Scalable Observation System (SOS) [20] is a framework for aggregating performance data from distributed workflows in support of in-situ analysis and feedback \& control. SOS is designed as an aggregation network consisting of client data sources, intermediate data listeners (one per allocation node), and one or more aggregation servers running on additional allocation nodes. Some HPC submission queues and batch systems don't allow launching multiple distributed applications per allocated node, so the SOS plugin follows a particular startup procedure to work around that limitation. The integration description below assumes that TAU is measuring an MPI application.

SOS is integrated into TAU as a plugin that can aggregate the data either as performance profiles or full event traces. In a typical usage scenario, the SOS plugin is configured to initialize the SOS client connection during TAU plugin initialization. It is assumed that one or more SOS aggregation daemons are already running on additional allocation nodes, having been launched by the submission script. If the SOS client library is unable to make a connection to an SOS listener daemon running on its node, the plugin will use the MPI infrastructure to coordinate between all ranks, group them ET node to launch an SOS listener daemon. The chosen rank will fork and exec the SOS listener process daemon for the SOS clients to connect to.

After the connections are established, the plugin will then optionally spawn a thread to perform asynchronous data aggregation over SOS. If asynchronous data aggregation isn't used, the data can be aggregated using the TAU trigger event. For profiling, the plugin 


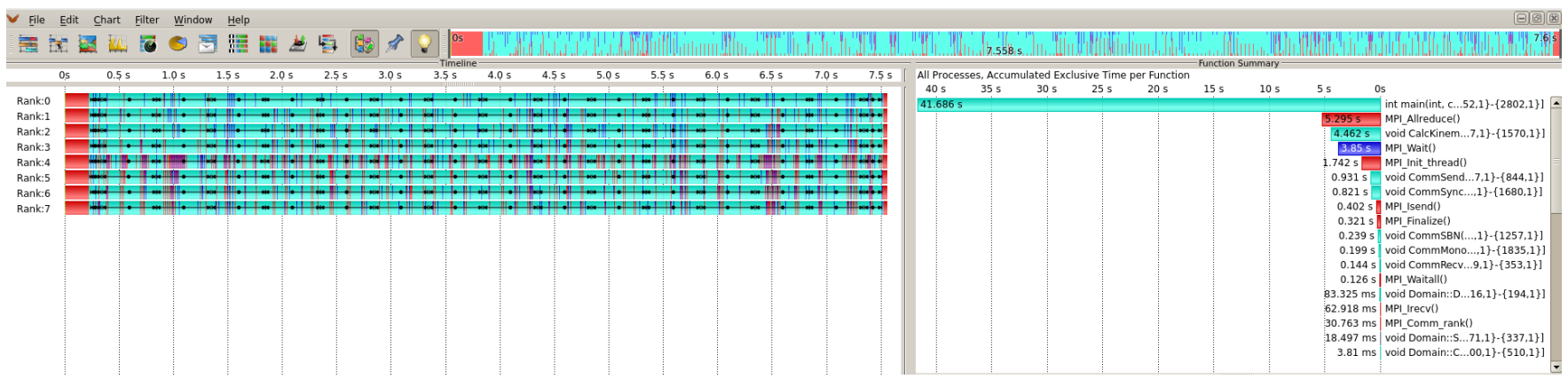

(a) Tracing all events

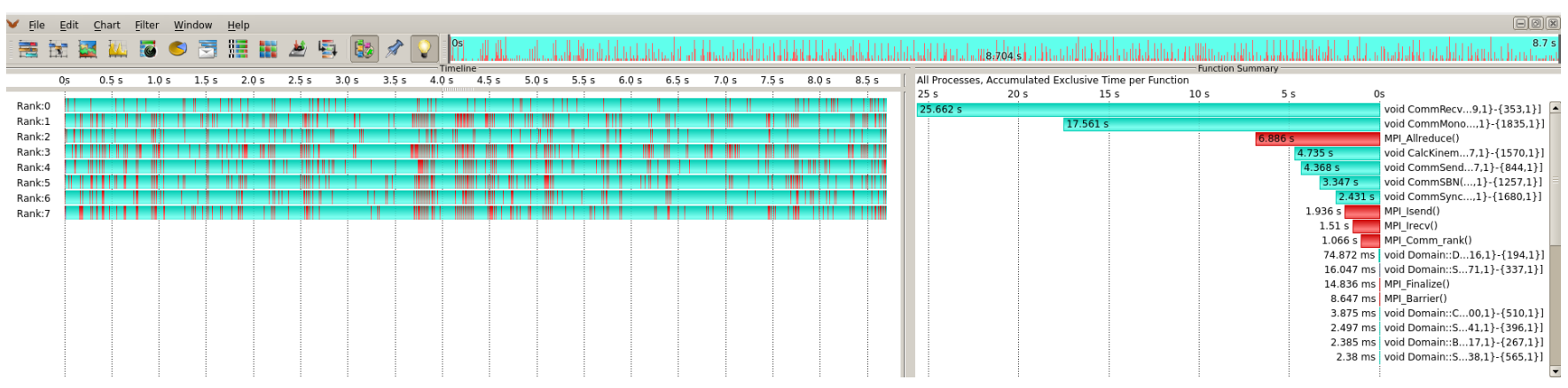

(b) MPI_Wait events disabled

Figure 6: Vampir: LULESH trace

code will iterate over the profile data of the current process, pack and publish it to the local listener. When tracing, the plugin will pack data in the local SOS client for each event and publish either periodically or on a trigger event. Once the listeners have received the data, they will then forward it to the aggregator(s).

\subsection{Trigger: Aggregating Interval Events}

The trigger mechanism is special in the sense that it allows an application to access TAU without an accompanying measurement being made. This can be harnessed to aggregate and snapshot performance information at an application-defined point of execution. We developed a plugin that aggregates and summarizes TAU interval and atomic event information across all threads of all processes inside the callback for the TRIGGER event. Note that this aggregation happens synchronously on the thread that invokes the trigger-event.

Additionally, the plugin depends on MPI to perform the necessary reduction operations on the TAU performance data. The summarized information is stored inside the plugin as a snapshot and can optionally be written out at the end of the application. The overheads involved in enabling this plugin depends on three factors:

- The number of processes involved in the reduction operation.

- Frequency of invocation of the trigger plugin.

- Number of interval and atomic events to be processed.

\subsection{Agent: Asynchronous Load Tracking}

An agent plugin is especially useful in a situation where a background task needs to be performed on a repetitive basis in such a way that the application execution isn't interrupted. Recall that the agent plugin is assigned a thread when the plugin is loaded. This thread can perform arbitrary tasks.

In addition to the SOS plugin that performs asynchronous work, we developed an agent plugin that periodically wakes up to record the current system load and resident set size in an OTF2 trace for every process. Specifically, the plugin creates a single pthread during the plugin initialization phase. In an MPI application, each MPI process would create its own agent thread. The load information is gathered from \proc \loadavg and the resident memory usage is extracted using the getrusage POSIX routine. Each of these metrics is internally stored as TAU user events and recorded in an OTF2 trace for offline visualization. The agent thread and its resources are cleaned up during the execution of PRE_END_OF_EXECUTION plugin state.

\section{EVALUATION}

In this section, we present a study of the performance of some of the plugins described in the section on Usage Scenarios. We pay special attention to the overheads involved in setting up and using multiple plugins together on the Intel Xeon Phi Knights Landing (KNL) processors. 


\subsection{Hardware and Software Setup}

Experiments on Intel KNL processors were performed on the Cori supercomputer ${ }^{1}$ at the National Energy Research Scientific Computing Center (NERSC). The KNL partition on Cori has a total of 9,688 compute nodes, where each node is a single-socket Intel Xeon Phi 7250 processor with 68 cores. Compute nodes are connected through the Cray Aries interconnect. The platform-optimized CrayMPICH was used on Cori.

6.1.1 LULESH. LULESH is a proxy application that represents a typical hydrocode such as ALE3D. LULESH is implemented using a variety of programming models. We use a version of LULESH that is parallelized using MPI and OpenMP. LULESH is a typical HPC simulation application - it consists of an outer iteration loop that runs for a fixed number of times specified by the input. The problem size scales with the number of MPI processes used. We instrumented LULESH using PDT to retrieve MPI as well as application-level information. We also added TAU annotation to trigger the TAU plugin module (using the TRIGGER plugin interface) at the end of every LULESH major iteration.

All the experiments in our study are run on 64 Intel Xeon Phi KNL nodes with pure MPI being used to parallelize LULESH. 64 processes of LULESH are executed per node for a total of 4096 MPI processes. The length of the cube mesh along each side is set to 60 , totaling 884,736,000 elements. Every experiment executes 500 iterations of the LULESH outer loop.

LULESH was executed using the selective tracing plugin that is described in Section 5.2 in combination with the trigger plugin (Section 5.5) that aggregates and snapshots TAU profile information globally at the end of every major iteration. The environment variable TAU_PLUGINS is used to specify the use of multiple plugins through the command line.

6.1.2 XSBench. XSBench is a mini-app that captures the core computational kernels within the larger OpenMC neutron transport code. XSBench is an MPI+OpenMP code. For our overhead studies, we used pure MPI to parallelize XSBench. We instrumented XSBench using PDT and TAU's PMPI wrapper.

The experiments for XSBench were run on 64 Intel Xeon Phi KNL nodes. 64 MPI processes were executed per node. We experimented with the small problem size with 34 lookups per particle for a total of 500,000 particles. The grid type was set to be unionized. XSBench was executed using the event counter plugin (Section 5.1) in combination with the load tracking agent plugin described in Section 5.6.

\subsection{Results}

An important aspect of our study is the overhead involved in enabling multiple plugins that perform non-trivial tasks. Table 2 depicts the overheads introduced by enabling the selective tracing and trigger-based profile aggregation plugins. Selective tracing is performed by disabling one or more events. For the study described in Table 2, tracing is enabled only for the MPI events. Meanwhile, all the events generated by PDT instrumentation are being profiled by TAU's measurement system. PDT instrumentation alone adds $1.4 \%$ of overhead over the baseline (un-instrumented) code.

\footnotetext{
${ }^{1}$ https://www.nersc.gov/users/computational-systems/cori/
}

Table 2: LULESH: Overheads with multiple plugins

\begin{tabular}{|l|c|}
\hline Run Description & Time (seconds) \\
\hline Default & 710 \\
PDT instrumentation & 720 \\
PDT + Tracing all events & 730 \\
PDT + Selective tracing & 730 \\
PDT + Selective tracing + Aggregation & 750 \\
\hline
\end{tabular}

Table 3: LULESH: Trace size with selective tracing

\begin{tabular}{|l|c|}
\hline Run Description & Trace Size (GB) \\
\hline Tracing all events & 6.6 \\
Tracing only MPI events & 5.5 \\
Tracing all events except MPI_Wait & 1.1 \\
\hline
\end{tabular}

The selective tracing plugin is responsible for an additional $1.4 \%$. Overall, the use of both plugins adds a total of $5.6 \%$ overheads over the baseline run. The trigger-based plugin aggregates 29 interval events and 2 atomic events during every LULESH iteration (for a total of 500 times).

Selective tracing is especially useful when one is trying to control the trace file size generated by a large parallel application. We measured the trace file sizes under the following conditions:

- Tracing all events.

- Selectively tracing only MPI events.

- Selectively tracing all MPI events except MPI_Wait events.

Table 3 clearly demonstrates that MPI is responsible for a bulk of the events in the trace. Specifically, MPI events account for $83.3 \%$ of the trace file size. Further, removing just the MPI_Wait class of events from the trace reduces the trace file size by $77.2 \%$ as compared to the default execution that includes all events.

For the XSBench application, we measured the overheads involved in enabling the event counter and load tracking agent plugins. Table 4 suggests that PDT instrumentation by itself does not add much overhead, but enabling the event counter plugin for all instrumented events leads to high overheads - over 100x in runtime. The sheer number of instrumented events (specifically, FUNCTION_ENTRY and FUNCTION_EXIT events) and the frequency of their invocation is a true stress test for our plugin architecture. Of course, we would not use this plugin in this manner. We reason that the source of the overhead is largely due to the hashing function for the strings representing function names.

However, when we used TAU's PMPI wrapper to instrument only the MPI events, the overheads are negligible. The lesson here is twofold. It is important to consider the impact of the number of events and their frequency when managing plugin overhead. The hashing logic needs to be optimized in the plugin framework. Interestingly, there are no noticeable overheads when the load tracking agent plugin is enabled. It is important to note that every MPI process spawns its own agent thread, accounting for a total of 64 agent threads per KNL node. This thread wakes up every 2 seconds to record the load and memory usage. 
Table 4: XSBench: Overheads with multiple plugins

\begin{tabular}{|l|c|}
\hline Run Description & Time (seconds) \\
\hline Default & 105 \\
PDT instrumentation & 106 \\
PDT + Event counter (all events) & $>100,000$ \\
PMPI wrapper + Event counter (only MPI events) & 106 \\
PMPI + Event counter (only MPI events) + Load tracking agent & 107 \\
\hline
\end{tabular}

\section{RELATED WORK}

Our ideas are inspired by research contributions in parallel performance systems, performance interfaces, measurement libraries, and performance monitoring frameworks. There are several remarkable performance systems developed for HPC applications, most notably HPCToolkit [13], Scalasca [14], Vampir [8], Score-P [12], Extrae [15], Open|SpeedShop [16], and TAU [1]. All are robust, scalable, and able to work with applications written in a variety of languages, targeting multiple models of parallelism, and executing in sophisticated runtime environments. It is almost certainly the case that these performance systems face the same challenges as TAU with respect to extending their architecture to extend functionality. Callbacks and plugins are powerful software techniques for adding new capabilities flexibly to systems in general.

It is easy to identify the usage of such techniques in how software and hardware interfaces are made accessible to performance measurement. The PMPI interposition concept in MPI and its extension with $P^{N} M P I$ [21] make possible the linking and activation of software based on an MPI call. Other interfaces are exposed for performance measurements using callback support, such as OpenMP OMPT [22] and CUDA CUPTI [23]. PAPI [24] implements statistical profiling by installing and emulating arbitrary callbacks on hardware counter overflow. These and other similar mechanisms are used by performance systems to be made aware of events taking place in libraries, runtime systems, and hardware, as well as to provide access to the context in which the events occurred.

The general notion of a producer-consumer model for performance tool interaction maps to examples where callbacks and plugins can be applied. One example is the RCRToolkit [25] which can gather information from various producers and provide a shared memory region for real-time access by consumers. It is used for resource-level observation to better understand the performance interactions in shared-memory systems. Similarly, the APEX [26] autonomic performance system provides asynchronous introspection of performance for policy-driven adaptive control. APEX was developed for the HPX runtime system to support dynamic task scheduling for performance and power optimization. Both RCRToolkit and APEX allow online performance data processing and support separation of concerns between components. While APEX does not implement a plugin model, it is based on an event/listener model (Observer design pattern), and functionality can be added to APEX by implementing additional listeners. Paradyn [27] is an early foundational system that captures the abstraction of a composable system with pluggable components addressing measurement and online analysis for performance discovery.

This perspective extends to performance monitors in general. Such is the case with the Periscope Tuning Framework [28]. Periscope enlists a communication and analysis agent framework that runs in a connected way with the application to identify performance problems at runtime. While the access to performance measurements takes place in a "monitor library" called by application processes, the actual analysis takes place in Periscope's analysis agent network running on separate processes. The AutoTune project [29] extended Periscope with plugin support for runtime control. It has been integrated with several parallel pattern libraries to tune parameters for power and scheduling.

There are two research projects closest to what we are discussing in this paper. The first concerns the extension of Score-P through plugins [30]. As noted above, Score-P [12] is a leading parallel performance system very similar to TAU. (In fact, TAU components can be integrated with Score-P.) It provides both "metric" plugins, which extend the standard timers and hardware counters, and "substrate" plugins, which enables custom processing of event streams, including doing analysis. In contrast, our plugin architecture works differently in that it allows a closer link between "states" and "triggers" while also allowing different plugins to be programmed and registered. We believe it would be possible to provide similar "metric" and "substrate" plugins within the TAU plugin architecture.

The second is the Caliper [31] performance system. Caliper consists of the source-code annotation API, a backend runtime component that manages blackboard buffers and the generalized context tree, add-on support services for control tasks (such as I/O and controlling snapshots), and additional data producer, measurement control, and data consumer services. Caliper utilizes a mixture of techniques identified in the tools, but combined in a novel and effective architecture. Snapshot mechanisms are the basic methods to collect data from data producers and provide it to data consumers. Data producers and consumers interact with Caliper through annotation and control APIs, or by registering callback functions for certain events. The callback interface provides notifications about system actions, measurement states, and event processing. Caliper is a newer performance system architecture than TAU and does not necessarily have the challenges TAU does. That said, there are similarities with respect to plugin support and it might be interesting to try to recreate certain Caliper functionality, such as the blackboards.

Finally, there is the interesting new support in PAPI for software defined events [32] that is related to our approach. The essential idea is that PAPI infrastructure can be extended to support events that come from different software layers (application, library, runtime) while leveraging the core PAPI infrastructures. These events are defined and programmed by the software developer. There is a way to register the events with PAPI, similar to plugin interfaces. Because the data created is all accessible within the PAPI system, performance systems that use PAPI, like TAU, could gain access. It would also appear that TRIGGER plugins could be used to reproduce PAPI software defined events.

\section{CONCLUSION AND FUTURE WORK}

To better address next-generation HPC performance analysis, advances in the TAU performance system are necessary to gain greater agility and adaptability in its operation. In this paper, we propose a TAU plugin architecture that will extend TAU's capabilities and open up possibilities for broader integration with complex parallel 
software environments. A prototype was produced to let us "test the waters" of the plugin architecture and experiment with different usage scenarios. The results are encouraging. TAU can now perform flexible measurement of parallel execution using STATE plugins than previously possible due to its constraints. TAU can execute TRIGGER plugins that are actuated directly by the application or other software and leverage direct acces to TAU's infrastructure for processing. The novel AGENT plugin gives TAU the capacity for asynchronous in situ measurement and analysis on additional resources devoted to that purpose. These plugins can be used in combination. Our Cori experiments give just a small glimpse of the potential the new plugin model might allow.

Of course, a prototype should not be released in the wild - the current TAU distribution is still what users have grown to love or hate. Before we do though, there are several aspects of the design and implementation that need further study and improvement. For instance, the plugin invocation overhead should be as efficient as possible and the filtering technique used for named events forces a string hash on every STATE plugin call. Furthermore, there are features that we have not yet fully explored, such as the ability of plugins to allocate and share memory and interact with each other through concurrent synchronized actions.

A strong motivation for our plugin efforts is driven by a desire to support runtime performance introspection, in situ performance analytics, and online adaptive tuning. The TAU performance system cannot pursue these opportunities to the full extent possible. Therefore, we intend to build upon the plugin framework to create stronger connections with scientific workflows, HPC data services, and in situ analysis and visualization environments.

\section{ACKNOWLEDGEMENT}

This work was supported by the NSF under the ACI-1450471 grant. This research used resources of the National Energy Research Scientific Computing Center (NERSC), a U.S. Department of Energy Office of Science User Facility operated under Contract No. DEAC02-05CH11231.

\section{REFERENCES}

[1] S. Shende and A. Malony. The tau parallel performance system. International Journal of High Performance Computing Applications, 20(2):287-311, 2006.

[2] A. Morris, A. Malony, S. Shende, and K. Huck. Design and Implementation of a Hybrid Parallel Performance Measurement System. September 2010.

[3] P. Mucci, S. Browne, C. Deane, and G. Ho. PAPI: A Portable Interface to Hardware Performance Counters. In DoD HPCMP Users Group Conference, pages 7-10, 1999.

[4] A. Knüpfer, R. Brendel, H. Brunst, H. Mix, and W. E. Nagel. Introducing the Open Trace Format (OTF). In Proceedings of the 6th International Conference on Computational Science, volume 3992 of Springer Lecture Notes in Computer Science, pages 526-533, Reading, UK, May 2006.

[5] K. Huck, A. Malony, R. Bell, and A. Morris. Design and Implementation of a Parallel Performance Data Management Framework. In 34th International Conference on Parallel Processing (ICPP). IEEE Computer Society, August 2005.

[6] R. Bell, A. Malony, and S. Shende. A Portable, Extensible, and Scalable Tool for Parallel Performance Profile Analysis. In European Conference on Parallel Processing (EuroPar), volume LNCS 2790, pages 17-26, September 2003.

[7] K. Huck and A. Malony. PerfExplorer: A Performance Data Mining Framework for Large-Scale Parallel Computing. In ACM/IEEE Conference on Supercomputing (SC). ACM, November 2005.

[8] Andreas Knüpfer, Holger Brunst, Jens Doleschal, Matthias Jurenz, Matthias Lieber Holger Mickler, Matthias S Müller, and Wolfgang E Nagel. The vampir performance analysis tool-set. In Tools for High Performance Computing, pages 139-155 Springer, 2008. www.vampir.eu.
[9] C. Eric Wu, Anthony Bolmarcich, Marc Snir, David Wootton, Farid Parpia, Anthony Chan, Ewing Lusk, and William Gropp. From trace generation to visualization: A performance framework for distributed parallel systems. In Proc. of SC2000: High Performance Networking and Computing, November 2000.

[10] F. Song, F. Wolf, N. Bhatia, J. Dongarra, and S. Moore. An Algebra for CrossExperiment Performance Analysis. In Proc. of International Conference on Parallel Processing, ICPP-04, August 2004.

[11] F. Wolf, B. Mohr, J. Dongarra, and S. Moore. Efficient Pattern Search in Large Traces through Successive Refinement. In Proceedings of the European Conference on Parallel Computing (EuroPar 2004, LNCS 3149), pages 47-54. Springer, 2004.

[12] et al. A. Knüpfer. Score-p: A joint performance measurement run-time infrastructure for periscope, scalasca, tau, and vampir. In 6th International Workshop on Parallel Tools for High Performance Computing, pages 79-91, 2012.

[13] et al. L. Adhianto. Hpctoolkit: Tools for performance analysis of optimized parallel programs. Concurrency and Computation: Practice and Experience, 22(6):685-701, April 2010.

[14] et al. M. Geimer. The scalasca performance toolset architecture. Concurrency and Computation: Practice and Experience, 22(6):702-719, April 2010.

[15] et. al P. Alonso. Tools for Power-energy Modelling and Analysis of Parallel Scientific Applications. In 41st International Conference on Parallel Processing (ICPP), pages 420-429, 2012.

[16] et al. M. Schulz. Open|SpeedShop: An Open Source Infrastructure for Parallel Performance Analysis. Scientific Programming, 16(2-3), April 2008.

[17] et. al S. Ramesh. MPI Performance Engineering with the MPI Tool Interface: Integration of MVAPICH and TAU. In 24th European MPI Users' Group Meeting (EuroMPI), 2017.

[18] Ian Karlin, Jeff Keasler, and JR Neely. Lulesh 2.0 updates and changes. Technical report, Lawrence Livermore National Lab.(LLNL), Livermore, CA (United States), 2013.

[19] Andreas Knüpfer, Holger Brunst, Jens Doleschal, Matthias Jurenz, Matthias Lieber, Holger Mickler, Matthias S Müller, and Wolfgang E Nagel. The vampir performance analysis tool-set. In Tools for High Performance Computing, pages 139-155. Springer, 2008.

[20] Chad Wood, Sudhanshu Sane, Daniel Ellsworth, Alfredo Gimenez, Kevin Huck, Todd Gamblin, and Allen Malony. A scalable observation system for introspection and in situ analytics. In 2016 5th Workshop on Extreme-Scale Programming Tools (ESPT), pages 42-49. IEEE, 2016

[21] M. Schulz and B. de Supinski. PNMPI Tools: A Whole Lot Greater than the Sum of Their Parts. In ACM/IEEE Conference on Supercomputing (SC), pages 30:1-30:10, 2007.

[22] et al. A. Eichenberger. Ompt: Openmp tools application programming interfaces for performance analysis. In International Workshop on OpenMP (IWOMP), pages 171-185, 2017.

[23] et. al A. Malony. Parallel Performance Measurement of Heterogeneous Parallel Systems with GPUs. In 40th International Conference on Parallel Processing (ICPP), pages 176-185, 2011.

[24] Philip J Mucci, Shirley Browne, Christine Deane, and George Ho. Papi: A portable interface to hardware performance counters. In Proceedings of the department of defense HPCMP users group conference, volume 710, 1999.

[25] Anirban Mandal, Rob Fowler, and Allan Porterfield. System-wide introspection for accurate attribution of performance bottlenecks. In Second International Workshop on High-perfromance Infrastruture for Scalable Tools, 2012.

[26] Kevin A Huck, Allan Porterfield, Nick Chaimov, Hartmut Kaiser, Allen D Malony, Thomas Sterling, and Rob Fowler. An autonomic performance environment for exascale. Supercomputing frontiers and innovations, 2(3):49-66, 2015.

[27] Barton P Miller, Mark D. Callaghan, Jonathan M Cargille, Jeffrey K Hollingsworth, R Bruce Irvin, Karen L Karavanic, Krishna Kunchithapadam, and Tia Newhall. The paradyn parallel performance measurement tool. Computer, 28(11):37-46, 1995.

[28] Shajulin Benedict, Ventsislav Petkov, and Michael Gerndt. Periscope: An onlinebased distributed performance analysis tool. In Tools for High Performance Computing 2009, pages 1-16. Springer, 2010.

[29] Renato Miceli, Gilles Civario, Anna Sikora, Eduardo César, Michael Gerndt, Houssam Haitof, Carmen Navarrete, Siegfried Benkner, Martin Sandrieser, Laurent Morin, et al. Autotune: A plugin-driven approach to the automatic tuning of parallel applications. In International Workshop on Applied Parallel Computing, pages 328-342. Springer, 2012.

[30] et al. R. Schöne. Extending the Functionality of Score-P through Plugins: Interfaces and Use Cases. In 10th International Workshop on Parallel Tools for High Performance Computing, page 59-82, October 2016.

[31] David Böhme, Todd Gamblin, David Beckingsale, Peer-Timo Bremer, Alfredo Gimenez, Matthew LeGendre, Olga Pearce, and Martin Schulz. Caliper: Performance introspection for hpc software stacks. In Proceedings of the International Conference for High Performance Computing, Networking, Storage and Analysis, SC '16, pages 47:1-47:11, Piscataway, NJ, USA, 2016. IEEE Press.

[32] A. Danalis, H. Jagode, T. Herault, P. Luszczek, and Jack Dongarra. Softwaredefined Events through PAPI. 2019. 